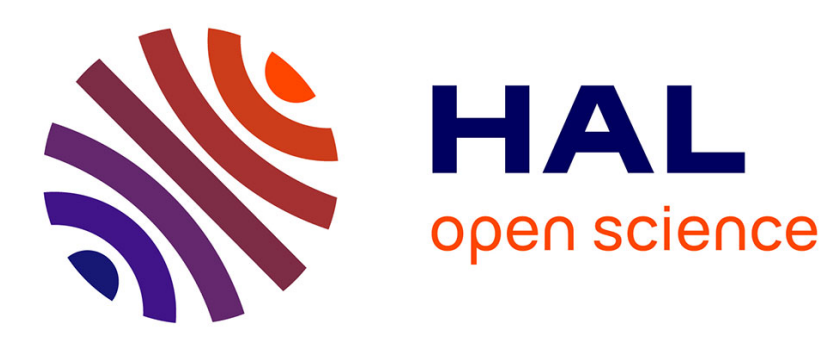

\title{
Réalisation d'une cellule d'absorption à réflexions multiples de grande dimension (longueur : $50 \mathrm{~m}$ )
}

\author{
J.P. Lux, A. Jenouvrier
}

\section{To cite this version:}

J.P. Lux, A. Jenouvrier. Réalisation d'une cellule d'absorption à réflexions multiples de grande dimension (longueur: $50 \mathrm{~m}$ ). Revue de Physique Appliquée, 1985, 20 (12), pp.869-875. 10.1051/rphysap:019850020012086900 . jpa-00245404

\section{HAL Id: jpa-00245404 https://hal.science/jpa-00245404}

Submitted on 1 Jan 1985

HAL is a multi-disciplinary open access archive for the deposit and dissemination of scientific research documents, whether they are published or not. The documents may come from teaching and research institutions in France or abroad, or from public or private research centers.
L'archive ouverte pluridisciplinaire HAL, est destinée au dépôt et à la diffusion de documents scientifiques de niveau recherche, publiés ou non, émanant des établissements d'enseignement et de recherche français ou étrangers, des laboratoires publics ou privés. 


\title{
Réalisation d'une cellule d'absorption à réflexions multiples de grande dimension (longueur : $50 \mathrm{~m}$ )
}

\author{
J. P. Lux et A. Jenouvrier \\ Laboratoire de Chimie Physique et Unité Associée au CNRS no 776(Spectrométrie Moléculaire et Atmosphérique), \\ U.E.R. Sciences, Moulin de la Housse, B.P. 347, 51062 Reims, France
}

(Reçu le 14 mai 1985, révisé le 8 août, accepté le 26 août 1985)

\begin{abstract}
Résumé. - Cet article décrit une cellule à réflexions multiples de grande dimension $(50 \mathrm{~m})$ parfaitement adaptée à des mesures de spectroscopie d'absorption en phase gazeuse. Sa conception et l'emploi d'une source de continuum suffisamment puissante rendent possibles des enregistrements continus avec des trajets optiques atteignant $2 \mathrm{~km}$ à $2000 \AA$ et $4 \mathrm{~km}$ à $2700 \AA$ (limités essentiellement par le pouvoir réflecteur des miroirs). Le dispositif expérimental, conçu principalement pour une utilisation dans l'UV $(\lambda>2000 \AA)$, est adapté aux cas où les sections efficaces d'absorption et (ou) les pressions de gaz utilisé sont faibles. Il a été testé sur l'oxygène et des résultats préliminaires aussi bien sur les bandes que sur le continuum de Herzberg sont présentés.
\end{abstract}

\begin{abstract}
We describe in this paper a $50 \mathrm{~m}$ long multiple reflection cell adapted to gaz phase absorption spectroscopic measurements. Its conception and the use of a continuum light source powerful enough allow continuous records with path lengths up to $2 \mathrm{~km}$ at $2000 \AA$ and $4 \mathrm{~km}$ at $2700 \AA$ (essentially limited by the reflecting power of the mirrors). The experimental device, first studied for use in the UV $(\lambda>2000 \AA)$ spectral range, is well adapted to the cases where the absorption cross sections and (or) the gaz pressures are small. Tests on oxygen absorption have been performed and preliminary results on the Herzberg bands and continuum are shown.
\end{abstract}

\section{Introduction.}

La spectroscopie d'absorption est un moyen très efficace de caractérisation et de mesure des constituants de l'atmosphère en utilisant le soleil ou les étoiles comme source lumineuse. Dans la «fenêtre» 2000 $2400 \AA$, l'opacité de l'atmosphère est contrôlée essentiellement par l'oxygène et l'ozone. (Continuum de Herzberg et de Hartley). Les mesures des sections efficaces d'absorption de ces deux espèces sont donc nécessaires à l'interprétation de la pénétration du flux solaire et des problèmes de photodissociation des constituants minoritaires $\left(\mathrm{N}_{2} \mathrm{O}\right.$, fréons...).

Actuellement, il existe une grande divergence $(30 \%)$ entre les mesures de laboratoire des sections efficaces de $\mathrm{O}_{2}$ et les mesures in situ de l'atténuation du flux solaire. Ce mauvais accord est généralement attribué à la mesure des sections efficaces de $\mathrm{O}_{2}$. Celles-ci étant très faibles $\left(\sigma \sim 10^{-23}\right.$ à $\left.10^{-24} \mathrm{~cm}^{2}\right)$, il est indispensable, pour pouvoir travailler au laboratoire dans des conditions de pressions proches des conditions atmosphériques $\left(p\left(\mathrm{O}_{2}\right)=1,8\right.$ torr à $30 \mathrm{~km}$ d'altitude), d'utiliser des trajets d'absorption très grands.
Ces conditions peuvent être obtenues au moyen d'une cellule à réflexions multiples que nous venons de construire. Etudiée en fonction de notre préoccupation présente, la mesure des sections efficaces d'absorption de l'oxygène dans l'UV, elle est conçue en vue d'une utilisation future dans toute région spectrale $\lambda>2000 \AA$ pour des mesures dans tous les cas où l'absorption et (ou) la pression de gaz à analyser sont faibles.

\section{Etude préliminaire.}

L'absorption dans une colonne de gaz est dans la plupart des cas régie par la loi de Beer Lambert qui peut s'écrire sous la forme

$$
\phi(\lambda)=\phi_{0}(\lambda) \exp \left(-\sigma(\lambda) N_{0} L \frac{P}{P_{0}} \frac{T_{0}}{T}\right)
$$

où $\phi_{0}(\lambda)$ et $\phi(\lambda)$ sont les intensités incidente et transmise dans une colonne de longueur $L, P_{0}$ et $T_{0}$ les pression et température standard, $P$ la pression du gaz à la température $T, N_{0}$ le nombre de Loschmidt 
$\left(2,687 \times 10^{19} \mathrm{~cm}^{-3}\right)$ et $\sigma(\lambda)$ la section efficace d'absorption.

Dans l'étude envisagée sur l'oxygène, $\sigma(\lambda)$ varie entre $10^{-23} \mathrm{~cm}^{2}$ à $2000 \AA$ et $10^{-24} \mathrm{~cm}^{2}$ à $2400 \AA$. Si on veut effectuer des mesures dans des conditions acceptables d'enregistrement, à savoir un minimum de $10 \%$ d'absorption $\left(\phi(\lambda) / \phi_{0}(\lambda)<0,9\right)$, il faut que le produit $P \times L$ soit supérieur à $3 \times 10^{3}$ et $3 \times 10^{4}$ torr $\times \mathrm{m}$ respectivement pour ces deux longueurs d'onde. Avec une pression de l'ordre de 10 torrs, les trajets d'absorption doivent donc atteindre 300 et $3000 \mathrm{~m}$ respectivement. Si $l$ est la longueur de la cellule et $n$ le nombre de réflexions, le trajet optique total est donné par $L=(n+1) l$. L'intensité du flux lumineux détectable par le système de mesure est $R^{n}$ fois l'intensité du flux incident où $R$ est le pouvoir réflecteur des miroirs. Si $R$ est faible $(<0,9)$, ce qui est le cas dans l'UV, le facteur $R^{n}$ décroît très vite avec $n$. Dans la région spectrale considérée, le problème est donc de trouver un compromis entre $l$ et $n$. Afin de garder une intensité suffisante, il est préférable d'augmenter la longueur de la cellule plutôt que le nombre de réflexions. Nous avons donc envisagé la construction d'une cellule de $50 \mathrm{~m}$ de longueur où un trajet de $2000 \mathrm{~m}$ est atteint pour 39 réflexions. Avec $R=0,9$, l'intensité du flux transmis est $\sim 1,6 \times 10^{-2}$ fois celle du flux incident, ce qui peut paraître très faible. L'utilisation d'une source lumineuse suffisamment puissante rend toutefois possible un enregistrement continu par détection photoélectrique.

\section{Le montage expérimental.}

Sur la figure 1, nous présentons le schéma de montage du dispositif expérimental. La source $S$ fournit le flux lumineux qui est focalisé par deux lentilles $L_{1}$ et $L_{2}$ à l'intérieur de la cellule au niveau du miroir A. La

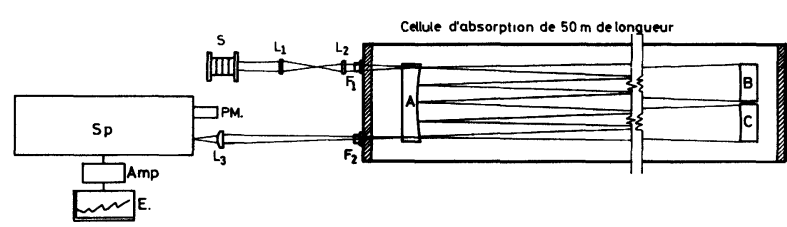

Fig. 1. - Schéma du montage expérimental. $\mathrm{S}$ : source de continuum : $\operatorname{arc} \operatorname{argon}, \mathrm{L}_{1}$ et $\mathrm{L}_{2}$ : lentilles biconvexes en suprasil, $L_{3}$ : lentille cylindrique en suprasil, $F_{1}$ et $F_{2}$ : fenêtres d'entrée et de sortie en suprasil, A, B, C : miroirs sphériques de $50 \mathrm{~m}$ de rayon de courbure assurant le système de réflexions multiples, $\mathrm{Sp}$ : spectromètre Jobin Yvon THR 1500, PM : photomultiplicateur, Amp : amplificateur, $\mathrm{E}$ : enregistreur.

[Experimental assembly diagram. $\mathbf{S}$ : continuum argon arc light source, $L_{1}$ and $L_{2}$ : biconvex suprasil lens, $L_{3}$ : cylindrical suprasil lens, $F_{1}$ and $F_{2}$ : entrance and exit suprasil windows, A, B, C : spherical $50 \mathrm{~m}$ radius of curvature mirrors for the multiple reflexion system, $\mathrm{Sp}$ : Jobin Yvon THR 1500 Spectrometer, PM : photomultiplier, Amp : amplifier, E : recorder.] longueur du trajet d'absorption est ajustée à l'aide des miroirs A, B et C. Le flux émergent est alors focalisé par une lentille cylindrique $\mathrm{L}_{3}$ sur la fente d'entrée du spectromètre équipé d'une chaîne de mesure composée d'un photomultiplicateur, d'un amplificateur et d'un enregistreur. Les lentilles $L$ et fenêtres $F$ sont en suprasil (coefficient de transmission $>90 \%$ à $2000 \AA$ ) afin de minimiser au maximum les pertes d'intensité dans l'ultraviolet.

3.1 LA CELlule À RÉFleXions MULTIPLes. - Élle peut être décrite en deux parties : l'enceinte proprement dite où est réalisé le vide et le système optique.

3.1.1 L'enceinte. - Elle est constituée en tube acier grade $B$ de $9 \mathrm{~mm}$ d'épaisseur et de $50 \mathrm{~cm}$ de diamètre intérieur. Ce tube (fourni par Vallourec Sedan) a été sablé au corindon afin d'obtenir une surface interne propre facilitant ainsi le dégazage. L'assemblage, effectué sur place par soudure bout à bout de cinq éléments (Serimer Villers Cotterets), a permis la réalisation d'un tube de $51 \mathrm{~m} 50$ de longueur. Chaque extrémité est fermée par des bouchons métalliques vissés et l'étanchéité assurée par des joints. Deux hublots disposés latéralement à chaque extrémité permettent d'observer la surface des miroirs. Des piquetages positionnés au centre du tube et à une extrémité sont prévus pour les prises de température, pression, introduction de gaz et remise à l'air libre de l'installation.

L'implantation d'un tel appareillage, à même le sol crayeux, dans un sous-sol de bâtiment partiellement thermostatisé de la faculté des Sciences, minimise au maximum les problèmes de vibration et dilatation probables au vu de la longueur du tube. Celui-ci repose sur des supports métalliques en $\mathrm{V}$ eux-mêmes solidaires de plots de béton coulés à intervalles réguliers directement dans le sol. La figure 2 présente une vue générale de l'installation en cours de montage.

Après une vérification très soigneuse de toutes les soudures par radiographie $\mathrm{X}$ et ressuage, la mise sous vide et le dégazage ont été obtenus au moyen d'un groupe de pompage installé dans une fosse au milieu du tube. Ce groupe, composé d'une pompe primaire biétagée D60A $\left(60 \mathrm{~m}^{3} / \mathrm{h}\right)$ et d'un roots WSU 251 $\left(250 \mathrm{~m}^{3} / \mathrm{h}\right)$ Leybold Heraeus Sogev, assure, à partir de la pression atmosphérique, l'évacuation de l'enceinte de $10 \mathrm{~m}^{3}$ en moins de 24 heures jusqu'à une pression résiduelle de $5 \times 10^{-4}$ torr.

3.1.2 Le système optique. - Le montage du système de miroirs assurant les réflexions multiples est basé sur l'idée originale de J. U. White [1]. Les miroirs A, B et C de même rayon de courbure sont positionnés de façon à ce que la distante $\mathrm{A}-(\mathrm{B}, \mathrm{C})$ soit exactement égale au rayon de courbure. Le rayonnement lumineux issu de la source $\mathrm{S}$, focalisé au niveau de la surface de $\mathrm{A}$, diverge pour couvrir $B$ puis est refocalisé sur $A$. L'ajustement de A est tel que son centre de courbure est situé entre $B$ et $C$, ceci entraîne que le faisceau réfléchi sur $\mathrm{A}$ couvre $\mathrm{C}$. La rotation de $\mathrm{B}$ et $\mathrm{C}$ autour 

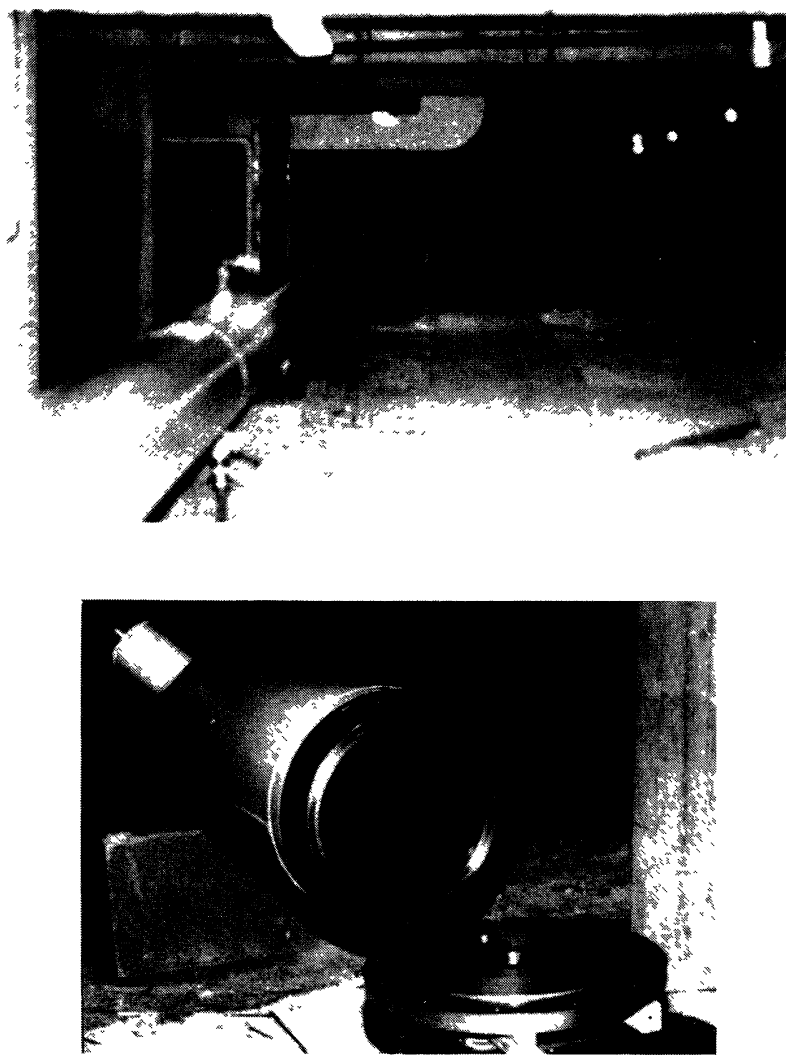

Fig. 2. - Vue générale de la cellule en cours de montage.

[Overall view of the cell during his assembly.]

de leur axe vertical permet le positionnement des images sur $\mathrm{A}$ et la sortie du faisceau.

Au lieu d'utiliser trois miroirs de même rayon de courbure difficiles à fabriquer car le rayon est très grand $(50 \mathrm{~m})$, nous avons préféré faire tailler puis découper (Fig. 3) un seul et même miroir suivant un schéma déjà utilisé $[2,3]$. Un maximum de fiabilité sur les rayons de courbure des trois parties $\mathrm{A}, \mathrm{B}$ et $\mathrm{C}$ est ainsi assuré tout en étant d'un prix de revient beaucoup moins élevé. Les deux encoches pratiquées dans la partie A permettent l'entrée et la sortie du faisceau lumineux. Les deux calottes $\mathrm{B}$ et $\mathrm{C}$ seront positionnées côte à côte en laissant un intervalle de $4 \mathrm{~mm}$ permettant leur rotation. Le polissage et le découpage ont été effectués par Reosc Optique avec les caractéristiques suivantes :

- Support : Zerodur Standard (verre de céramique) ayant un coefficient de dilatation très bas $(0 \pm 0,15 \times$ $\left.10^{-6} \mathrm{~K}^{-1}\right)$.

- Rayon de courbure : $5000 \pm 15 \mathrm{~cm}$.

- Diamètre poli : $40 \mathrm{~cm}$.

- Epaisseur : 7,5 cm.

- Qualité de surface : définie par l'angle entre la normale à la surface réelle et celle de la meilleure sphère inférieure en tout point à 4 " d'arc.

La couche réfléchissante choisie et déposée par Matra Optique est un revêtement classique aluminium

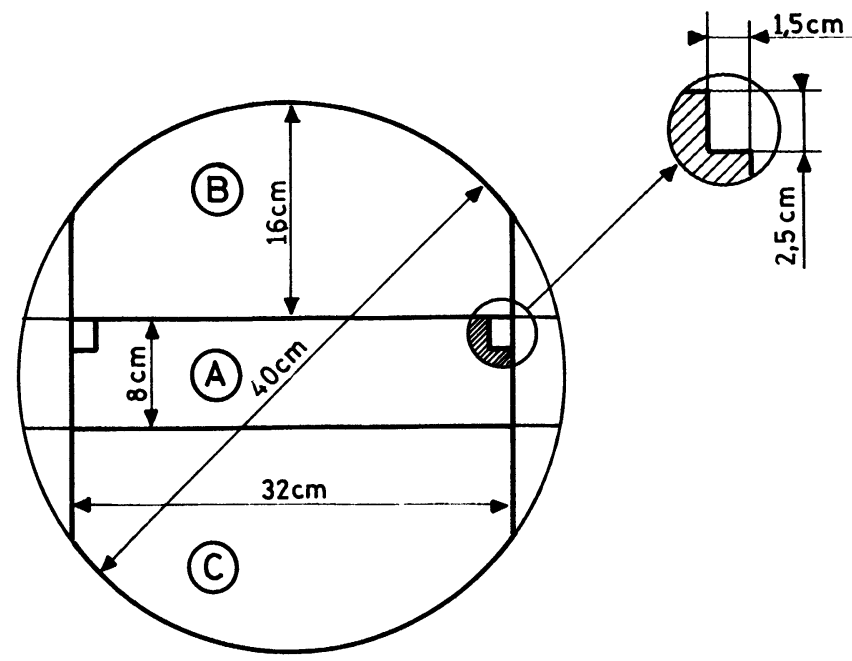

Fig. 3. - Schéma de découpe des miroirs.

[Mirrors cuting diagram.]

protégé par un dépôt $\mathrm{MgF}_{2}$ assurant un pouvoir réflecteur $>80 \%$ pour $\lambda>2000 \AA$ et pouvant être utilisé jusque dans l'infrarouge.

- Les miroirs avec leurs supports sont montés à l'intérieur du tube sur des plaques d'embase en acier réglables sur des tiges filetées verticales traversant le tube et fixées dans des plots de béton. L'étanchéité au vide est assurée par des tombacs. Ce montage permet de s'affranchir des problèmes de dilatation de l'enceinte et donc de minimiser les risques de désalignement du système optique. Les déplacements des miroirs (rotation, inclinaison), aussi bien pour le réglage que pour le changement du nombre de réflexions sont assurés par des platines et vis différentielles Microcontrôle que nous avons motorisées en adaptant sur chaque élément des motoréducteurs MDP. Ceux-ci sont commandés de l'extérieur de la cellule via des passages de courant fixés sur les bouchons de fermeture des extrémités du tube. La commande électrique se fait à partir d'un même boîtier, ce qui permet à une même personne de régler facilement le système optique sans se déplacer.

La figure 4 présente plusieurs vues de la mécanique de commande. On peut y remarquer que les déplacements et l'orientation sont assurés par :

- Une platine de rotation et un berceau goniométrique pour le miroir $\mathrm{A}$.

- Une platine de déplacement linéaire et des vis différentielles pour les miroirs $\mathbf{B}$ et $\mathbf{C}$.

La mise au point de la distance entre miroirs (focus) est obtenue par translation du miroir A sur une glissière visible également sur les photographies.

Des mouvements de très faible amplitude peuvent ainsi être obtenus et les images sur le miroir A positionnés avec une grande précision $(<1 \mathrm{~mm})$. La rotation d'un miroir B ou C d'un angle $\alpha=2 \times$ $10^{-4}$ rad déplace l'image sur A de $1 \mathrm{~mm}$. 

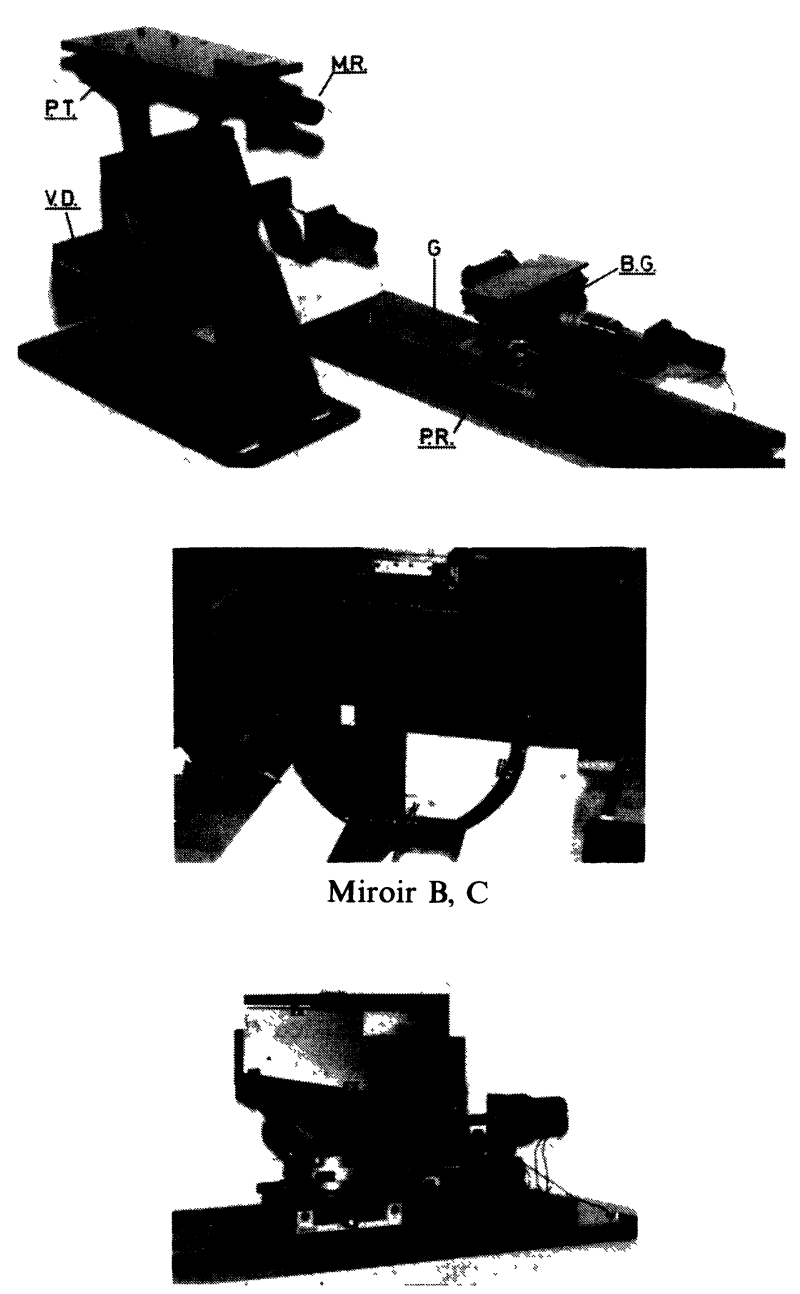

Miroir A

Fig. 4. - Mécanique de commande des miroirs. BG : berceau goniométrique, $\mathrm{PR}$ : platine de rotation, $\mathrm{G}$ : glissière, PT : platine de translation, VD : vis différentielles, MR : motoréducteur.

[Mirrors control mechanics. BG : goniometrical cradle, PR : rotation plate, $\mathrm{G}$ : slide, $\mathrm{PT}$ : translation plate, VD : differential screws, $\mathrm{MR}$ : motoreductor.]

3.2 LA SOURCE De CONTINUUM. - Mise au point à l'Institut National de Métrologie et commercialisée par Drusch, elle est constituée (Fig. 5a) d'un arc électrique sous pression atmosphérique d'argon ayant une puissance variant de 0,2 à $1,7 \mathrm{~kW}$. Le plasma fournit un spectre continu entre 1150 et $3300 \AA$. L'arc est alimenté en gaz par un débit continu d'argon de l'ordre de 2 à 4 litres/min. La longueur et le diamètre du plasma sont respectivement de $8 \mathrm{~mm}$ et $4 \mathrm{~mm}$. Opérant sous une tension de 30 à $35 \mathrm{~V}$ avec un courant pouvant varier de 5 à $50 \mathrm{~A}$, l'arc fournit un flux dont la luminance spectrique est représentée sur la figure $5 \mathrm{~b}$. La courbe correspondante d'une lampe classique à deutérium $(20 \mathrm{~W})$ donnée à titre de compa-

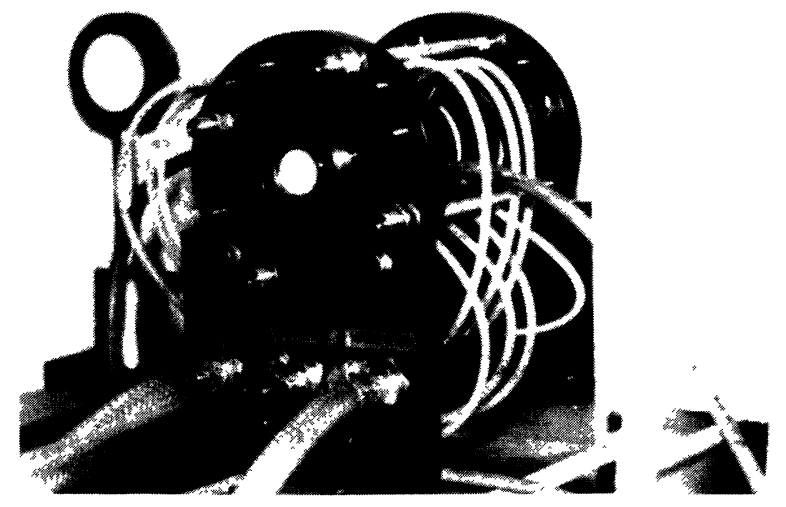

a)

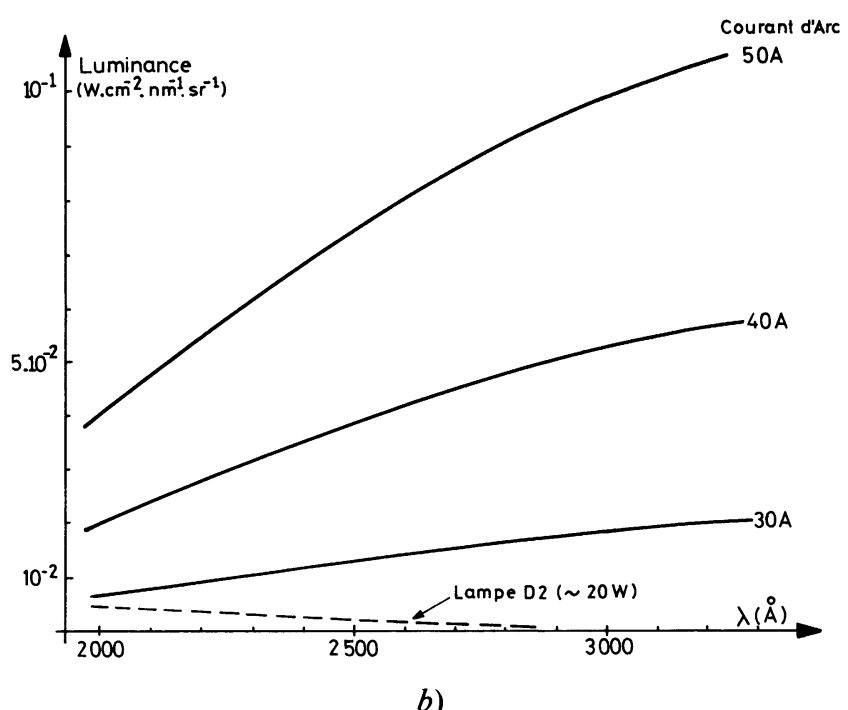

Fig. 5. - Arc argon et luminance spectrique entre 2000 et $3300 \AA$.

[Argon arc light source and spectric luminance between 2000 and $3300 \AA$. .]

raison permet de se rendre compte de la puissance de l'arc.

3. 3 LE SYSTÈME DE DÉTECTION. - Le flux émergent de la cellule est focalisé sur la fente d'entrée d'un monochromateur Jobin Yvon THR 1500 ayant les caractéristiques suivantes :

Distance focale : $1,5 \mathrm{~m}$ - Ouverture : $f / 12$.

Réseau holographique 2400 traits $/ \mathrm{mm}$.

Dispersion : 2,6 $\AA / \mathrm{mm}$ - Résolution : 150000 en simple passage.

La chaîne de mesure est constituée actuellement par :

Photomultiplicateur Hamamatsu R928 ou R955.

Amplificateur continu.

Enregistreur Philips PM 8222.

La saisie des données sera effectuée simultanément dans peu de temps par un microordinateur Apple II. 


\section{Performances et mesures.}

Après le montage et la mise en place des miroirs sur leurs supports respectifs, l'alignement et la mise au point du système optique de réflexions multiples ont été réalisés en suivant le trajet lumineux d'un laser hélium néon. Son faisceau est légèrement divergent. Entrant au niveau de A, il donne une image d'environ $5 \mathrm{~cm}$ de diamètre sur le miroir $B$ situé à $50 \mathrm{~m}$ simulant ainsi les conditions d'utilisation avec la source $\mathrm{S}$. Les performances de l'appareillage ont alors été testées avec l'arc argon.

\section{1 Performances.}

4.1.1 Focalisation - Astigmatisme - Trajet optique. - Les lentilles $\mathrm{L}_{1}$ et $\mathrm{L}_{2}$ forment de la source une image de $1 \mathrm{~cm}$ de diamètre au niveau du miroir $\mathrm{A}$. La focalisation du système, très difficile à déterminer est obtenue par déplacement du miroir A sur sa glissière en surveillant l'évolution de la qualité des images (intensité et non grandissement) quand on augmente le nombre de réflexions. Les conditions les meilleures sont obtenues pour une distance entre les miroirs de $5006 \pm 2 \mathrm{~cm}$ correspondant aux normes fournies par le constructeur sur le rayon de courbure $(5000 \pm 15 \mathrm{~cm})$.

Compte tenu des dimensions des miroirs, l'ouverture du système est d'environ $f / 320$. Avec cette faible valeur, les problèmes liés à l'astigmatisme sont tout à fait négligeables. Un calcul rapide (cf. Reesor [2]) montre que l'élargissement par astigmatisme de l'image après 40 réflexions n'excède pas $0,001 \mathrm{~cm}$.

Le miroir A ayant une longueur de $32 \mathrm{~cm}$, il semble difficile de pouvoir positionner plus de 30 images sur une même horizontale (taille des images $1 \mathrm{~cm}$ ). Le trajet optique maximum possible est donc $6 \mathrm{~km}$. Dans la pratique, celui-ci est limité par le pouvoir réflecteur. Nous avons pu toutefois enregistrer sans difficultés le flux de la source avec des trajets de 2 et $4 \mathrm{~km}$ à 2000 et $2700 \AA$. En fait, les miroirs ayant un rayon de courbure de 50,06 m et la surface du miroir A étant située à $80 \mathrm{~cm}$ des fenêtres d'entrée et de sortie de la cellule(Fig. 1) les trajets optiques exacts sont $2003,0 \mathrm{~m}$ et $4006,4 \mathrm{~m}$.

4.1.2 Pouvoir réflecteur - Diffusion. - Le pouvoir réflecteur des miroirs a été mesuré en étudiant la perte d'intensité du flux lumineux due au nombre croissant de réflexions. La courbe de la figure 6 présente, dans le domaine spectral $2000-2800 \AA$, la variation de $R$ (pouvoir réflecteur), exprimée en pourcentage, en fonction de la longueur d'onde. Légèrement inférieur à $80 \%$ pour $\lambda=2000 \AA, R$ atteint $92 \%$ à $2800 \AA$. Dans ce domaine, $R$ reste supérieur à ce que garantit le constructeur. Pour des longueurs d'onde supérieures, la croissance régulière de $R$ doit permettre d'atteindre des trajets optiques plus grands.

La qualité de la surface des miroirs peut être évaluée en mesurant la lumière diffusée par la cellule. Pour ce

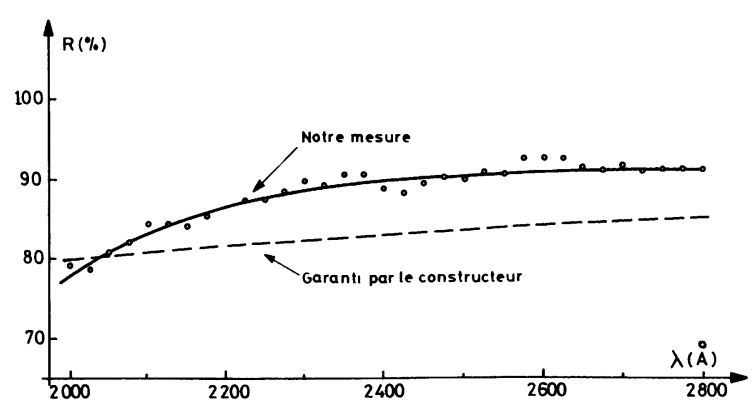

Fig. 6. - Pouvoir réflecteur des miroirs (revêtement $\left.\mathrm{Al}+\mathrm{MgF}_{2}\right)$.

[Reflecting Power of the mirrors ( $\mathrm{Al}+\mathrm{MgF}_{2}$ coating).]

faire, le faisceau de sortie est déplacé légèrement de manière à ce que seule la lumière non focalisée puisse sortir de l'enceinte. Avec un trajet optique de $1000 \mathrm{~m}$, le taux de lumière diffusée est inférieur à $0,2 \%$ dans le domaine $2000-2400 \AA$.

4.1.3 Stabilité et bruit de source. - En spectroscopie d'absorption, la mesure d'une intensité est conditionnée par un paramètre très important qui est la stabilité de la source de fond continu. Dans notre montage, la source utilisée s'est révélée très stable. Après un temps de chauffage nécessaire à la mise à température du plasma d'argon, l'enregistrement du flux lumineux émis ne laisse pas apparaître de dérive supérieure à $0,5 \%$ après plusieurs heures de fonctionnement.

La reproductibilité est meilleure que $1 \%$ après de nombreux allumages successifs. Le bruit de photons sur le détecteur, fonction de l'intensité enregistrée reste inférieur à $2 \%$ de l'intensité du flux total.

4.1.4 Enregistrements. - De nombreux tests ont pu être effectués dans le domaine spectral 2 000-2 $400 \AA$ en enregistrant, « cuve vide ", l'intensité du flux lumineux. Un exemple est donné sur la figure 7 pour des trajets variant de 1200 à $2000 \mathrm{~m}$ dans le domaine spectral 2 100-2 $275 \AA$. Pour un même trajet, la décroissance en intensité vers les plus courtes longueurs d'onde est liée à celle de la source elle-même, aux pertes par transmission des éléments optiques (lentilles $L$, fenêtres $F$, réseau) et à la sensibilité spectrale du photomultiplicateur. Entre des trajets différents, l'atténuation est directement fonction du pouvoir réflecteur des miroirs.

4.2 Mesures : ABSORPTION DE L'OXYGÈne. - Des mesures préliminaires à notre travail sur la détermination des sections efficaces de l'oxygène dans le domaine spectral $2000-2400 \AA$ ont été réalisées afin de tester notre appareillage. Nous présentons les résultats obtenus dans deux domaines différents correspondant à deux types de spectres, un discret $(\lambda>2420 \AA)$ et un continu $(\lambda<2420 \AA)$. 


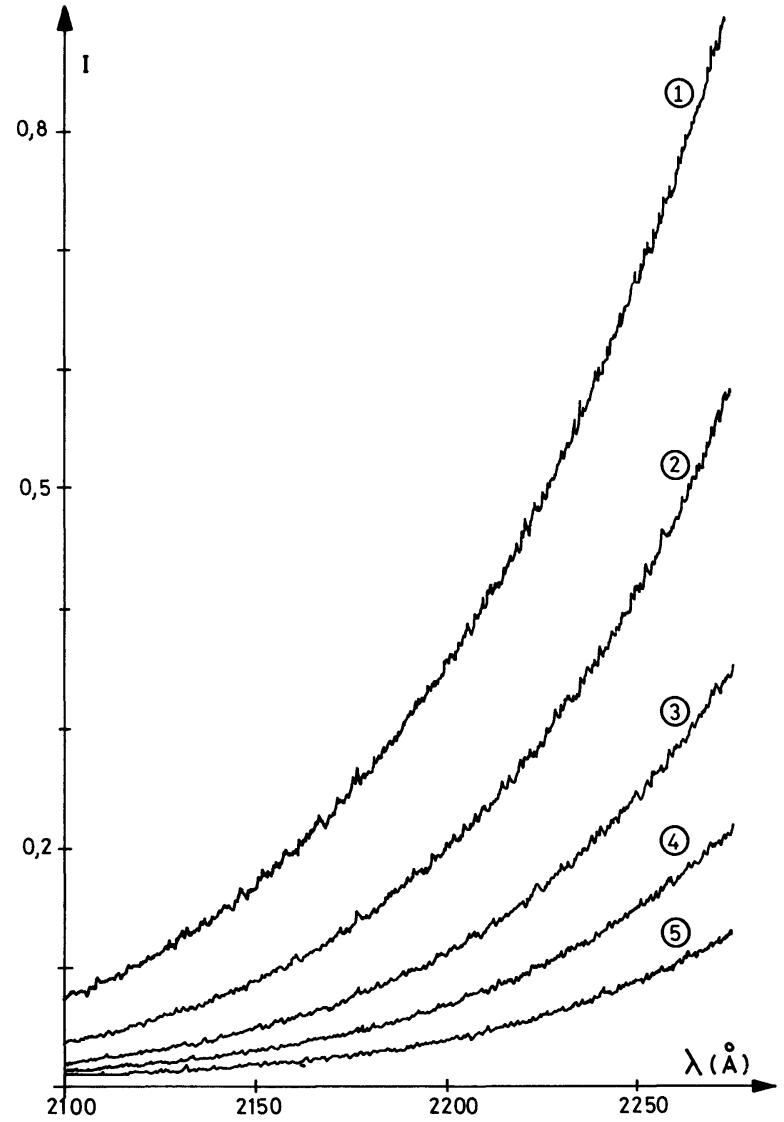

Fig. 7. - Intensité du continuum pour différents trajets optiques dans le domaine spectral 2 100-2 $275 \AA$.

[Continuum light source intensities for differents optical path lengths in the $2100-2275 \AA$ spectral range.]

Trajets d'absorption. [Path lengths.] : (1) $1203,04 \mathrm{~m}$, (2) $1403,28 \mathrm{~m}, 31603,52 \mathrm{~m}$, (4) $1803,76 \mathrm{~m}$, (5) $2004,00 \mathrm{~m}$.

- Pour $\lambda>2420 \AA$, l'oxygène présente un spectre d'absorption discret dont la contribution majeure est apportée par le système $\mathrm{A}^{3} \Sigma_{\mathrm{u}}^{+}-\mathrm{X}^{3} \Sigma_{\mathrm{g}}^{-}$étudié par Herzberg [4]. La figure 8 montre un enregistrement dans le domaine 2410-2 470 $\AA$ des bandes d'absorption 9-0, 10-0 et 11-0 de ce système qui se dissocie vers $2420 \AA$. Bien visibles sur la courbe b), les raies $Q$ de ces bandes apparaissent déjà sur la courbe a) $\left(p\left(\mathrm{O}_{2}\right) \sim 0\right)$. Ceci correspond à l'absorption de l'oxygène à l'extérieur de la cuve sur le trajet entre la source et la cuve et entre la cuve et le détecteur soit environ $7 \mathrm{~m}$. Le produit $P \times L$ dont dépend l'intensité d'absorption est $7 \times \frac{760}{5} \simeq 1,06 \times 10^{3}$ torr $\times \mathrm{m}$ alors que pour la courbe b), il est $8,52 \times 10^{4}$ torr $\times \mathrm{m}$.

- Pour $\lambda<2420 \AA$, l'oxygène absorbe d'une manière continue (continuum de Herzberg). Aucune structure n'est visible et les courbes de la figure 9 montrent bien que l'absorption en fonction de la longueur d'onde est monotone pour une pression déterminée de gaz. Dans ce domaine, les difficultés de mesures tiennent au fait que les sections efficaces

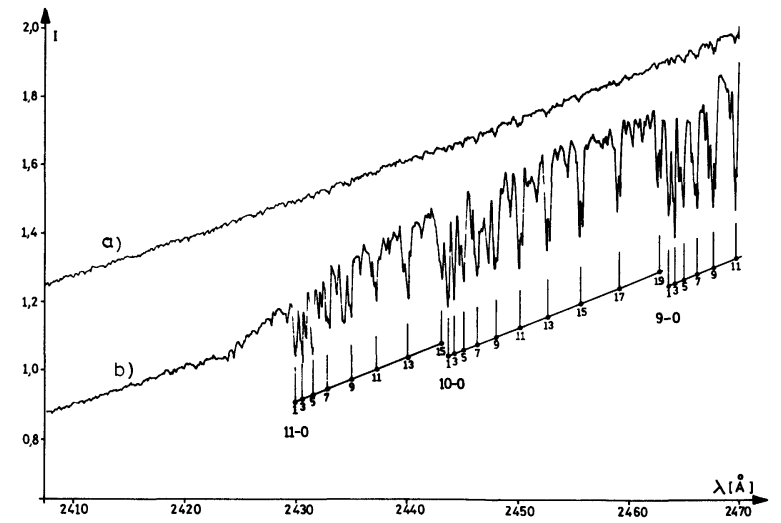

Fig. 8. - Absorption de l'oxygène dans le domaine spectral $2410-2470 \AA$ montrant les bandes 9-0, 10-0 et 11-0 du système $A-X$ ainsi que la limite de dissociation vers $2420 \AA$. (Les raies $\mathrm{Q}$ sont identifiées par leur valeur de $N$ ). a) $p\left(\mathrm{O}_{2}\right)=0$ b) $p\left(\mathrm{O}_{2}\right)=85$ torrs. Conditions expérimentales : trajet optique $1002,8 \mathrm{~m}$, défilement spectromètre $5 \AA / \mathrm{mm}$, défilement enregistreur $20 \mathrm{~mm} / \mathrm{min}$, fente $35 \mu$, largeur de bande : 0,09 $\AA$.

[Oxygen absorption tracing in the $2410-2470 \AA$ spectral range showing the 9-0, 10-0 and 11-0 bands of the A-X system and the dissociation limit near $2420 \AA$. (The $Q$ lines are labelled with their $N$ values). a) $p\left(\mathrm{O}_{2}\right)=0$; b) $p\left(\mathrm{O}_{2}\right)=$ 85 torrs. Experimental conditions : path length $1002.8 \mathrm{~m}$, continuous wavelength scan speed $5 \AA / \mathrm{mm}$, recorder speed $20 \mathrm{~mm} / \mathrm{min}$, slit width $35 \mu$, band width $0.09 \AA$.]

d'absorption sont faibles (de $10^{-24}$ à $\left.10^{-23} \mathrm{~cm}^{2}\right)$ et dépendent de la pression : $\sigma=\sigma_{0}+\sigma_{1} \cdot P$ où $\sigma_{0}$ est la section efficace de $\mathrm{O}_{2}$ à pression nulle et $\sigma_{1}$ une section efficace généralement attribuée à la formation de $\mathrm{O}_{4}$. Les mesures antérieures de laboratoire (Hasson et Nicholls [5], Ditchburn et Young [6], Ogawa [7], Shardanand et Rao [8], Johnston et al. [9]) présentent une grande divergence due certainement à une mauvaise détermination de $\sigma_{0}$ par extrapolation (trajet d'absorption trop faible et donc pression trop élevée). Les résultats de la figure 9 sont traduits par les valeurs de $\sigma_{0}$ sur la figure 10 et comparés à ceux des auteurs ci-dessus à $\lambda=2250 \AA$. A cette longueur d'onde, notre valeur de $\sigma_{0}$ est plus basse de $5 \%$ seulement que celle des références $[6,8,9]$. La courbe $\sigma_{0}=f(\lambda)$ doit être confirmée par d'autres enregistrements afin de mesurer le degré de reproductibilité des résultats en variant les conditions expérimentales. Il est néanmoins permis de penser que ce résultat préliminaire confirme le bon fonctionnement général du système de mesure. Cependant, deux problèmes importants doivent encore être pris en compte pour une détermination absolue de $\sigma_{0}$ de $\mathrm{O}_{2}$ :

- Les phénomènes de diffusion au niveau du réseau du spectromètre, généralement assez importants dans l'ultraviolet, mesurables en intercalant des filtres interférentiels.

La diffusion Rayleigh dans la cuve elle-même. 


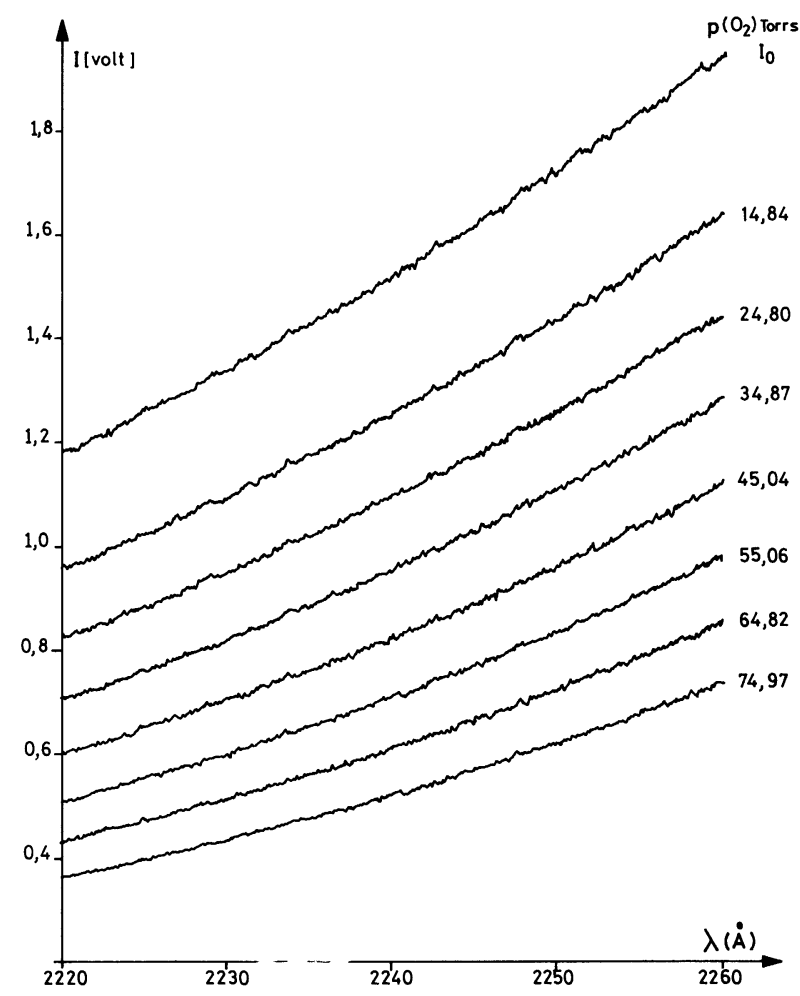

Fig. 9. - Absorption de l'oxygène dans le continuum de Herzberg pour différentes pressions. Conditions expérimentales : domaine spectral $2220-2260 \AA$, trajet optique $1002.8 \mathrm{~m}$, défilement spectromètre $5 \AA / \mathrm{mm}$, défilement enregistreur $20 \mathrm{~mm} / \mathrm{min}$, fente $96 \mu$, largeur de bande $0,25 \AA$.

[Oxygen absorption tracing in the Herzberg continuum with different pressions. Experimental conditions : spectral range : $2220-2260 \AA$, path length $1002,8 \mathrm{~m}$, continuous wavelength scan speed $5 \AA / \mathrm{mm}$, recorder speed $20 \mathrm{~mm} / \mathrm{min}$, slit width $96 \mu$, band width $0.25 \AA$.]

\section{Conclusion.}

Le principe de la cellule à réflexions multiples étant connu depuis longtemps, la description précédente a pour but principal de faire connaître l'existence et les caractéristiques de notre appareil. Actuellement, il en existe peu de cette taille et à notre connaissance, le modèle que nous venons de construire est un des plus

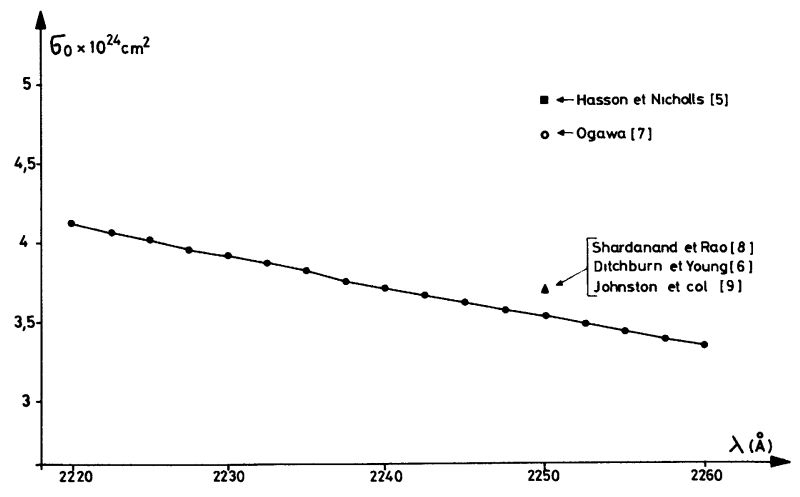

Fig. 10. - Sections efficaces d'absorption de $\mathrm{O}_{2}$ dans le domaine spectral $2220-2260 \AA$.

$\left[\mathrm{O}_{2}\right.$ absorption cross sections in the $2220-2260 \AA$ spectral range.]

performants. Les trajets optiques qu'il permet d'atteindre font de celui-ci un outil très efficace pour l'étude fondamentale des molécules par absorption. Optimisé dans un premier temps pour des études dans l'ultraviolet, il peut sans problèmes particuliers être utilisé jusque dans l'infrarouge. Les performances actuelles pourront ultérieurement être encore améliorées par l'utilisation d'une meilleure détection (Transformée de Fourier par exemple) et l'augmentation des trajets optiques en doublant le nombre d'images (cf. Horn et Pimentel [3]) dans les régions où le pouvoir réflecteur est suffisamment grand. Le dispositif actuel est cependant assez performant pour pouvoir réaliser les mesures des sections efficaces d'absorption de $\mathrm{O}_{2}$ qui est le but prioritaire de nos recherches actuelles.

\section{Remerciements.}

Ce présent travail se situe dans lę cadre d'une étude : "La pénétration du rayonnement solaire dans l'atmosphère. Mesure des sections efficaces d'absorption de $\mathrm{O}_{2}$ et $\mathrm{O}_{3}$ entre 2000 et $2400 \AA$ ». Il n'aurait pu être mené à bien sans le soutien du CNRS et du Ministère chargé de l'Environnement que les auteurs tiennent particulièrement à remercier.

\section{Bibliographie}

[1] White, J. U., J. Opt. Soc. Am. 32 (1942) 285.

[2] ReEsor, T. R., J. Opt. Soc. Am. 41 (1951) 1059.

[3] Horn, D. et Pimentel, G., Appl. Opt. 10(8) (1972) 1892.

[4] Herzberg, G., Can.J. Phys. 30 (1952) 185.

[5] Hasson, V. et Nicholls, R. W., J. Phys. B 4 (1971) 1789.

[6] Ditchburn, R. W. et Young, P. A., J. Atmos. Terr. Phys. 24 (1962) 127.
[7] Ogawa, M., J. Chem. Phys. 54(6) (1971) 2550.

[8] Shardanand et Prasad Rao, A. D., J. Quant. Spectrosc. Radiat. Trans. 17 (1977) 433.

[9] Johnston, H. S., PAIGE, M. et YAO, F., J. Geophys. Res. 89 (1984) 11661. 